MATEC Web of Conferences 3, 01019 (2013)

DOI: $10.1051 /$ matecconf/20130301019

(c) Owned by the authors, published by EDP Sciences, 2013

\title{
Temperature-resolved second harmonic generation: a tool designed to study solid-solid transition
}

\author{
S. Clevers, F. Simon, V. Dupray, and G. Coquerel
}

PRES Normandie, Crystallogenesis Unit, SMS, EA 3233 IMR 4114 Université de Rouen, F-76821 Mont Saint-Aignan Cedex, France

\section{Introduction}

The second harmonic generation (SHG) is a nonlinear optical process occurring only in noncentrosymmetric space groups. The measurement of the SHG signal generated by powdered samples [1] is an extremely sensitive tool for the detection of noncentrosymmetric phases at the ppm level [2] and was already used successfully to detect the noncentrosymmetry of crystal arrangements such as conglomerates [3]. Recently, we developed a set-up for temperature resolved SHG (TRSHG) in order to monitor solid-solid transitions. In this communication, new possibilities of this technique for the characterization of organic compounds in the solid state are presented. In particular, the desolvation of lactose and the solid-solid phase transition of organic compounds (involving metastable equilibrium) are discussed.

\section{Experimental}

\subsection{Temperature Resolved Second Harmonic Generation (TR-SHG)}

Figure 1 shows the experimental setup used for the TRSHG measurements. A Nd:YAG Q-switched laser (Quantel) operating at $1.06 \mu \mathrm{m}$ was used to deliver 360 $\mathrm{mJ}$ pulses of $5 \mathrm{~ns}$ duration with a repetition rate of $10 \mathrm{~Hz}$. An energy adjustment device made up of two polarizers (P) and a half-wave plate $(\lambda / 2)$ allowed to vary the incident energy from 0 to ca. $200 \mathrm{~mJ}$ per pulse. A RG1000 filter was used after the energy adjustment device to remove light from laser flash lamps. The samples (few mg of powder in a crucible) were placed in a computer controlled Heating-Cooling stage (Linkam THMS-600) and were irradiated with a beam (diameter of $4 \mathrm{~mm})$.

The signal generated by the sample (diffused light) was collected into an optical fibre $(500 \mu \mathrm{m}$ of core diameter) and directed onto the entrance slit of a spectrometer (Ocean Optics).

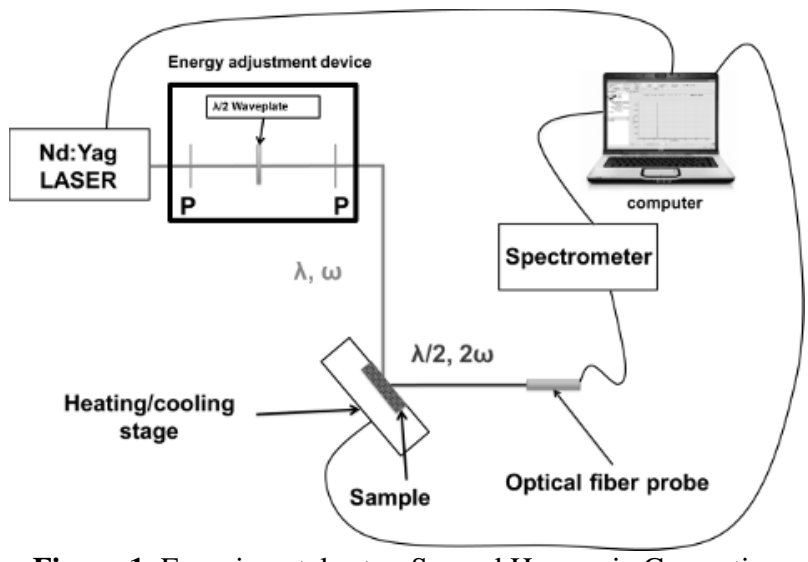

Figure 1. Experimental setup Second Harmonic Generation Apparatus constituted of Nd:YAG Q-switched laser operating at $1064 \mathrm{~nm}$.

A boxcar integrator allowed an average spectrum (spectral range $490-590 \mathrm{~nm}$ ) with a resolution of $0.1 \mathrm{~nm}$ to be recorded over $2 \mathrm{~s}$ (20 pulses). In non-suitable cases, to avoid problems related to the sublimation of the samples, the heating-stage was opened but only during the SHG measurements so it had no significant influence on the temperature regulation.

According to Kurtz and Perry SHG powder method [1], SHG signal intensities were compared to the signal of a reference compound (quartz - $45 \mu \mathrm{m}$ average size).

\subsection{Differential Scanning Calorimetry (DSC)}

Thermal analyses of the solids were conducted on DSC 204 F1 Netzsch equipped with an Intracooler. Solid samples (mass of c.a $15 \mathrm{mg}$ with maximum deviation of $0.05 \mathrm{mg}$ ) were placed in a $25 \mu \mathrm{L}$ close aluminum crucible. The atmosphere of the analyses was regulated by Helium flux (40 mL.min $\left.{ }^{-1}\right)$, and heat runs were conducted at different constant heating rate. The data treatment was performed with the Netzsch-TA Proteus $₫$ Software v 4.8.4. 


\section{Results}

\section{Solid-Solid phase transition}

\section{3-Hydroxybenzoic acid (MHBA)}

MHBA exhibits two polymorphic forms (a metastable and a stable form) which have been described in literature $[4,5]$. Metastable form of MHBA crystallizes as an orthorhombic structure in the non-centrosymmetric space group $P$ na $2{ }_{1}$. Thus, it exhibits SHG activity. The stable form crystallizes as a monoclinic structure in the centrosymmetric space group $P 2_{1} / \mathrm{n}$ (SHG inactive). Conversion of metastable MHBA into stable crystal form was monitored by TR-SHG. This phase transition involves metastable equilibrium; therefore a TTT diagram (not shown) was established from isothermal TR-SHG measurements. Moreover, the activation energy of the solid-solid phase transition was determined and compared to values obtained from DSC analysis.

\section{Pyrazinamide}

TR-SHG of commercial pyrazinamide $\left(P 2_{1} / \mathrm{c} \quad-\right.$ centrosymmetric space group) was performed at 5 K.min 1 (Figure 2). Pyrazinamide exhibits a relatively high vapor pressure above $140{ }^{\circ} \mathrm{C}$. The commercial pyrazinamide have no SHG signal because of centrosymmetric crystal structure. The SHG signal increases strongly at circa $145{ }^{\circ} \mathrm{C}$ due to the recrystallization (after sublimation) of a new phase of pyrazinamide in the non-centrosymmetric space group $(P \mathrm{c})$.

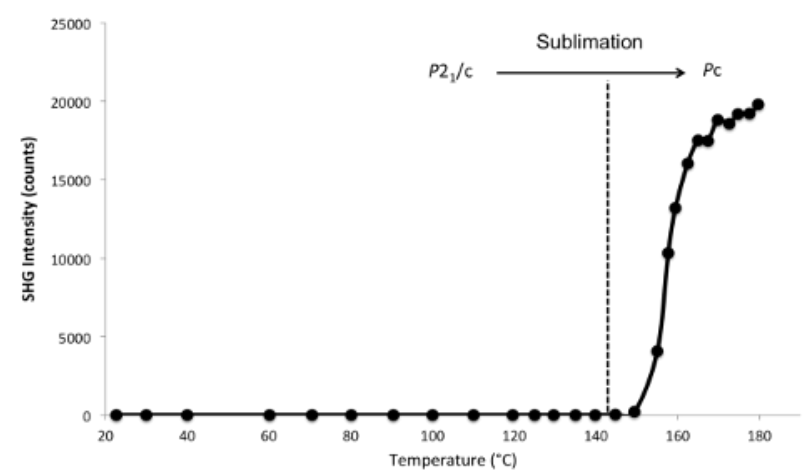

Figure 2. SHG intensity versus temperature $\left(5 \mathrm{~K} \cdot \mathrm{min}^{-1}\right.$ heating rate). Vertical dashed line corresponds to the starting temperature of sublimation detected by SHG

\section{Desolvation of $\alpha$-lactose monohydrate $(\mathrm{L} \alpha-\mathrm{H} 2 \mathrm{O})$}

DSC analysis for a heating rate of $5 \mathrm{~K} \cdot \mathrm{min}^{-1}$ was performed on commercial $\alpha$-lactose monohydrate hereafter $\mathrm{L} \alpha-\mathrm{H}_{2} \mathrm{O}$ (purchased from Acros Organics, CAS number: 5989-81-1) (Figure 3). Dehydration can be clearly identified on the thermogram by the endothermic peak (with shoulder) at the onset temperature of 139.1 ${ }^{\circ} \mathrm{C}$. This thermal event is followed by an exothermic peak at $155.9{ }^{\circ} \mathrm{C}$ associated to a conversion into stable anhydrous phase [6,7].

TR-SHG was also performed on $\mathrm{L} \alpha-\mathrm{H}_{2} \mathrm{O}$. The TRSHG curve (SHG intensity versus temperature) is plotted on Figure 3.

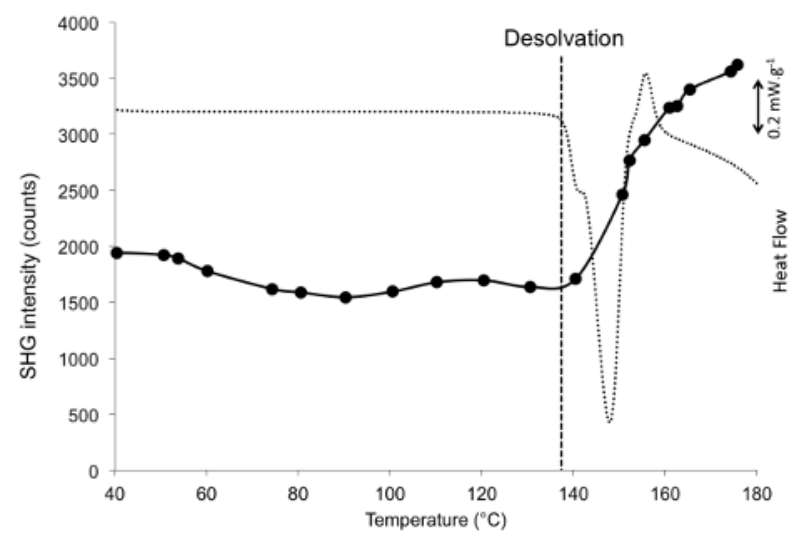

Figure 3. SHG intensity versus temperature (circle) and DSC chart (dash line). Vertical dash line indicates the temperature of desolvation.

Results clearly show a slope disruption at circa $140{ }^{\circ} \mathrm{C}$ that is correlated to the desolvation of the monohydrate phase toward the anhydrous phase. Then SHG appears even more suitable to track the desolvation of $\mathrm{L \alpha}-\mathrm{H}_{2} \mathrm{O}$ than it is sufficiently accurate to detect a slight change in the crystalline parameters (the anhydrous phase formed is most likely the isomorph desolvate which belongs to the same space group as the monohydrate). Note that because of the degradation of the sample, the SHG signal decreases for temperature upper than $190^{\circ} \mathrm{C}$ (result not shown).

\section{Conclusion}

We show that TR-SHG can be used: (i) to determine the activation energy of solid-solid transitions, (ii) to study the stability of phases, (iii) to study desolvation mechanisms.

Throughout these the various cases (involving compounds with noncentrosymmetric structures), we demonstrate that TR-SHG can be an appropriate tool to detect and monitor phase transitions in the solid state.

\section{References}

1. S.K. Kurtz, T.T. Perry, J. Appl. Phys. 393798 (1968)

2. S. Clevers, F. Simon, V. Dupray, G. Coquerel, Journal of Thermal Analysis and Calorimetry (2012) DOI: $10.1007 / \mathrm{s} 10973-012-2763-y$

3. A. Galland, V. Dupray, B. Berton, S. Morin-Grognet, M. Sanselme, H. Atmani, G. Coquerel, Crystal Growth \& Design 92713 (2009)

4. G.V. Gridunova, N.G. Furmunova, Y.T. Struchkov, Z.I. Ezhkova, L.P. Grigoreva, B.A. Chayanov, Kristallografiya 27267 (1982)

5. F.L. Nordström, Å.C. Rasmuson , European Journal of Pharmaceutical Sciences 28377 (2006)

6. S. Garnier, S. Petit, G. Coquerel, Journal of Thermal 
Analysis and Calorimetry 68489 (2002)

7. S. Garnier, S. Petit, F. Mallet, M.N. Petit, D.

Lemarchand, S. Coste, J. Lefebvre, G. Coquerel,

International Journal of Pharmaceutics 361131 (2008) 